\title{
Observation of Phase Transition of Liquid Crystalline Polymers by Ultrasonic Method. (Hydroxypropyl)cellulose and ( $n$-Hexanoyloxypropyl)cellulose
}

\author{
Akira TanaKa, * Yorihisa Hamada, Hiroshi OnOda, \\ Takanari YamaguCHI, and Koh-hei NitTa \\ Department of Polymer Chemistry, Kyoto University, Kyoto 606, Japan.
}

(Received October 4, 1993)

\begin{abstract}
Using ultrasonic measurements, phase transition behavior was examined for (hydroxypropyl)cellulose (HPC) in solution and ( -hexanoyloxypropyl)cellulose (HPC-C6) in bulk. For various kinds of HPC solutions, we observed a phase transition of Landau-Khalatnikov type in the variation of ultrasonic variables with polymer concentration. It is the first observation for lyotropic liquid crystalline polymer systems, so far as we know. The critical concentration varied with species of solvent and it became higher in the order of acetic acid, ethyl alcohol, dioxane, and water. For HPC-C6 in bulk, we found at least two kinds of phase transitions in the variation of ultrasonic variables with temperature. One of them is ascribable to the phase transition between liquid crystalline and isotropic phases (i.e., the phase transition of the first kind). Another phase transition may be one of the second kind, in the case in which one could not detect by conventional methods. Thus, it was found that ultrasonic method is very useful to study the phase transition behavior.
\end{abstract}

KEY WORDS Ultrasonic Velocity / Propagation Intensity / Phase Transition / Landau-Khalatnikov Transition / (Hydroxypropyl)cellulose / (nHexanoyloxypropyl)cellulose / Lyotropic Liquid Crystalline Polymer / Thermotropic Liquid Crystalline Polymer /

(Hydroxypropyl)cellulose (designated as HPC) forms a liquid crystalline phase in a variety of aqueous and organic solvents ${ }^{1-4}$; HPC exhibits lyotropic liquid crystalline characteristics. Formation of ordered phase is observed for most soluble cellulosic polymers ${ }^{5-7}$ and it is presumably related to the lack of flexibility of the cellulose backbone. The chirality of the anhydroglucose repeat unit promotes formation of cholesteric mesophase; the order in the liquid crystal is characterized in terms of a helicoidal supermolecular arrangement.

The introduction of flexible branches is known to enhance the mobility of cellulosic polymers and promotes an ordered phase in bulk..$^{8-13}$ For instance, ( $n$-hexanoyloxypropyl)cellulose (designated as HPC-C6) forms a cholesteric liquid crystalline phase in bulk in a particular range of temperature; HPC-C6 exhibits thermotropic liquid crystalline characteristics. The branches may play a role like a solvent.

For liquid crystalline polymer systems, several phases appear: crystal phases, different types of liquid crystalline phases, isotropic liquid phase, and so on. Therefore, there exist several phase transitions between different phases. Phase transition can be classified in the following two kinds ${ }^{14}$; one is the phase transition of the first kind, at which order parameter becomes zero discontinuously and

\footnotetext{
* To whom correspondence should be addressed.
} 
thermodynamic functions such as volume and entropy change discontinuously. The other is the phase transition of the second kind, at which order parameter becomes zero continuously and thermodynamic functions change continuously.

The phase transition behavior has been examined by conventional methods such as optical microscope observation and DSC measurement. However, one can hardly detect the phase transition of the second kind using such conventional methods. It is also difficult to detect the phase transition of the first kind when the thermodynamic functions such as volume and enthalpy change a little. In this study, we introduced an ultrasonic method. Ultrasonic variables are expected to response sensitively to the fluctuation or the relaxation of order parameter, ${ }^{15}$ and consequently to the phase transitions of the first and second kinds. ${ }^{16}$

In this study, we measured ultrasonic properties of HPC and HPC-C6 in their phase transition region. In the case of $\mathrm{HPC}$, ultrasonic properties were measured in solution as a function of concentration (i.e., in the process of solvent evaporation). In the case of HPC-C6, on the other hand, they were measured in bulk as a function of temperature.

Besides, optical microscope observation and DSC measurement were carried out to help our understanding of ultrasonic data. Furthermore, visual observation of cholesteric colors was done as a function of concentration or temperature. The cholesteric structure consists of rotating layers of rodlike molecules $^{17,18}$; it is characterized by the presence of a helical twist with uniform pitch. The helicoidal supermolecular arrangement provides striking optical properties to the solution. For instance, the circularly polarized light is reflected at a wavelength related to the pitch of the helicoidal arrangement. When the cholesteric pitch is comparable to the wavelength of visible light, one can see beautiful cholesteric reflection colors. De Vries theory ${ }^{19}$ predicts that beautiful colors are due to the selective reflection of the visible light; the selective reflection is caused by uniform cholesteric twisted structure.

\section{EXPERIMENTAL}

\section{Preparation and Characterization of Samples}

(Hydroxypropyl)cellulose, HPC-SL produced by Nippon Soda Co., Ltd. was used. According to Horio et $a l,{ }^{20}$ the weight average molecular weight $\left(M_{w}\right)$ of this polymer is 164300 , the number average molecular weight $\left(M_{n}\right) 41600$, the degree of molar substituent (MS) 3.5, and the degree of substituent (DS) 2.3.

We prepared (n-hexanoyloxypropyl)cellulose (HPC-C6) by esterification of HPC $^{21,22}$; first, HPC of $30 \mathrm{mg}$ dried in a vacuum for a week was dissolved in dry acetone of $750 \mathrm{ml}$ previously treated by zeolite. Into the solution, pyridine of $30 \mathrm{mg}$ was added and then $n$ hexanoyl chloride of $100 \mathrm{mg}$ was added drop by drop. The adding was achieved in an icewater bath. After completion of adding, the solution was refluxed at $60-70^{\circ} \mathrm{C}$ for about three hours. Then, HPC-C6 thus obtained were precipitated in methyl alcohol. The precipitate was furthermore purified by repeating of dissolution and precipitation; acetone was used for dissolution and methyl alcohol for precipitation.

IR spectra were measured on HPC and HPC-C6 using Perkin Elmer Model 1600 FT-IR spectrophotometer. Comparing the spectrum of HPC-C6 with that of HPC, the following differences were seen; (1) The peak intensity at $3500 \mathrm{~cm}^{-1}(\mathrm{O}-\mathrm{H}$ stretching band) decreased. (2) The peak newly appeared at $1725 \mathrm{~cm}^{-1}$ ( $\mathrm{C}=\mathrm{O}$ stretching band). (3) The peak intensity among 1100 and $1500 \mathrm{~cm}^{-1}$ (C-H bending of alkyl group) increased. Proton NMR spectra of HPC and HPC-C6 were also measured using JEOL FT-270 NMR spectrophotometer $(270 \mathrm{MHz})$. A few differences were seen for the spectrum of HPC-C6; 
(1) The peak intensity in the high magnetic field $(\delta=0.9-2.5 \mathrm{ppm})$ caused by the introduction of alkyl group increased. (2) The peak of propyl proton shifted to the lower magnetic field $(\delta=5.0 \mathrm{ppm})$. (3) The peak intensity of hydroxy proton $(\delta=2.7-4.5 \mathrm{ppm})$ decreased. Thus, esterification was verified by the IR and NMR spectra. The degree of substitution (DS) of ester group was also determined from proton NMR spectra ${ }^{23}$ and it was about 2.6; otherwise, almost the same value was obtained independently from the elementary analysis.

Thermal properties of HPC-C6 were examined on the heating and cooling runs using Perkin Elmer DSC-7. Measurements were carried out in the temperature range between -50 and $170^{\circ} \mathrm{C}$. Both the heating and the cooling rates were $20^{\circ} \mathrm{C} \mathrm{min}^{-1}$. Only one peak was observed on the thermogram of heating and cooling runs, respectively. On the heating run an endothermic peak was observed at temperatures from 100 to $150^{\circ} \mathrm{C}$ and its maximum temperature was around $128^{\circ} \mathrm{C}$. On the cooling run an exothermic peak was observed at temperatures from 132 to $110^{\circ} \mathrm{C}$ and its maximum temperature was around $125^{\circ} \mathrm{C}$. In either case, the peak was very faint and the enthalpy change was about 1 joule per gram.

\section{Procedure of Ultrasonic Measurement}

HPC solution of $20 \mathrm{wt} \%$ was first prepared. The following four solvents were used: distilled water, ethyl alcohol, acetic acid, and dioxane. Then, the solution was poured in a teflonmade cell as shown in Figure 1. Using the cell, ultrasonic properties of HPC solutions were measured in the process of solvent evaporation. On occasion, we wrapped the cell in polyvinylidene fluoride film so that solvent molecules evaporate as gently and uniformly as possible. Four transducers are fixed at the bottom of the cell. One of transducers converts an electric impulse into an impulsive mechanical vibration. Such an impulsive ultrasonic wave travels through the sample and then it is

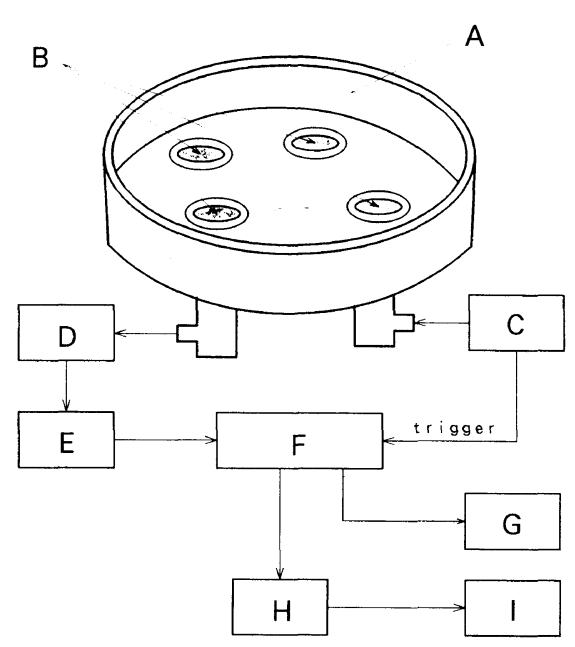

Figure 1. The illustration of a device for ultrasonic measurements in the process of solvent evaporation. A, cell; $\mathrm{B}$, transducer; $\mathrm{C}$, function generator; $\mathrm{D}$, differential preamplifier; E, discriminator; F, transient memory; G, oscillator; H, computer; I, printer.

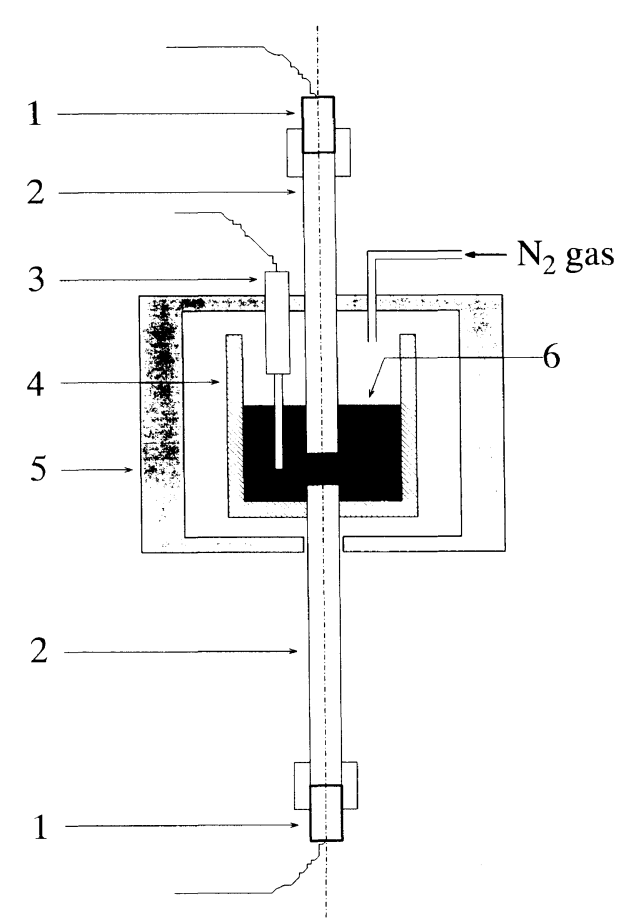

Figure 2. The illustration of a device for ultrasonic measurements in the heating and cooling processes. 1, transducer; 2, waveguide; 3 , thermometer; 4, cell; 5, heating box; 6 , sample. 
converted into electrical signal by another transducer again. Consequently, one can measure ultrasonic properties of solutions at six different distances (from 1.4 to $3.8 \mathrm{~cm}$ ). The resonant frequency of transducers used was $150 \mathrm{kHz}$. The concentration of solution was determined by the total weight of cell and solution.

Ultrasonic properties of HPC-C6 were measured on heating and/or cooling runs using an apparatus shown in Figure 2. Heating was achieved by circulating $\mathrm{N}_{2}$ gas heated by electric heat band and cooling by sending vapor of liquid $\mathrm{N}_{2}$ at an appropriate rate. Waveguides ( $\# 2$ ) made of stainless steal were inserted between sample $(\# 3)$ and transducer $(\# 1)$ to avoid the damage of transducers at high temperatures. Also in this case, the measurements were done by an impulsive ultrasonic wave method using transducers with a resonant frequency of $150 \mathrm{kHz}$.

The ultrasonic velocity $(v)$ was determined from the propagation time $(\Delta t)$ and the distance between transducers $(L)$ using the following equation.

$$
v=\frac{L}{\Delta t}
$$

The propagation intensity $(A)$ is expressed as in the following equation,

$$
A=A_{0} \mathrm{e}^{-\alpha L}
$$

where $\alpha$ is the attenuation coefficient and $A_{0}$ the ultrasonic intensity at $L=0$. The equation is rewritten as follows.

$$
-\ln A=-\ln A_{0}+\alpha L
$$

Hence, $-\ln \mathrm{A}$ corresponds to the attenuation coefficient when $A_{0}$ and $L$ are constant.

\section{RESULTS AND DISCUSSION}

\section{Ultrasonic Properties of HPC in the Process of Solvent Evaporation}

Figure 3 shows the variation of ultrasonic velocity $v$ and $-\ln A$ with concentration for

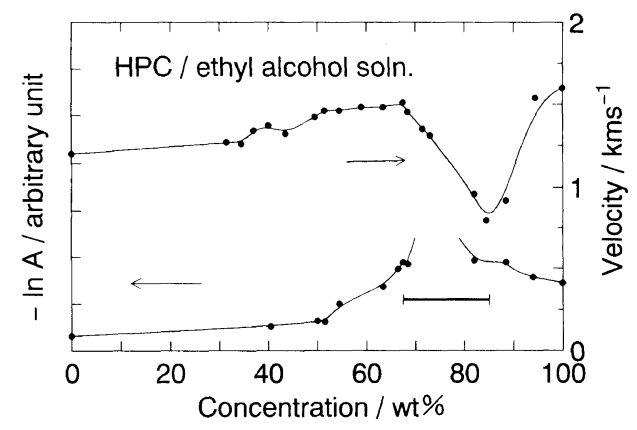

Figure 3. The variation of ultrasonic velocity $v$ and $-\ln A$ ( $A$ is the amplitude of ultrasonic wave at a given distance) with concentration for ethyl alcohol solution of HPC.

ethyl alcohol solution of HPC. The value of $-\ln A$ began to increase around $50 \mathrm{wt} \%$ and diverged around $75 \mathrm{wt} \%$. Above $80 \mathrm{wt} \%$ it decreased again. The ultrasonic velocity, on the other hand, began to decrease around 70 $w t \%$. After it became lowest around $85 \mathrm{wt} \%$, it increased again with increasing concentration. The solid bar in the figure denotes the concentration range in which iridescent colors were observed. The concentration range $(68-85 \mathrm{wt} \%)$ is almost coincident with the range in which the velocity decreased and $-\ln$ $A$ changed divergently.

The concentration dependence of ultrasonic properties of other HPC solutions prepared from different solvents was also investigated. The results are shown in Figures 4 and 5 . Figure 4 shows the variation of velocity with concentration and Figure 5 that of $-\ln A$. Solid bars in the figures also indicate the concentration range in which iridescent colors were observed. The following observation is in common for all solutions; the concentration range in which the velocity changes anomaly is almost the same as the propagation intensity does. Also, it is quite the same as iridescent colors are observed. The iridescent colors are caused by regular cholesteric pitches as mentioned above. Therefore, it was concluded that the anomalous change in the velocity and the propagation intensity is ascribable to 


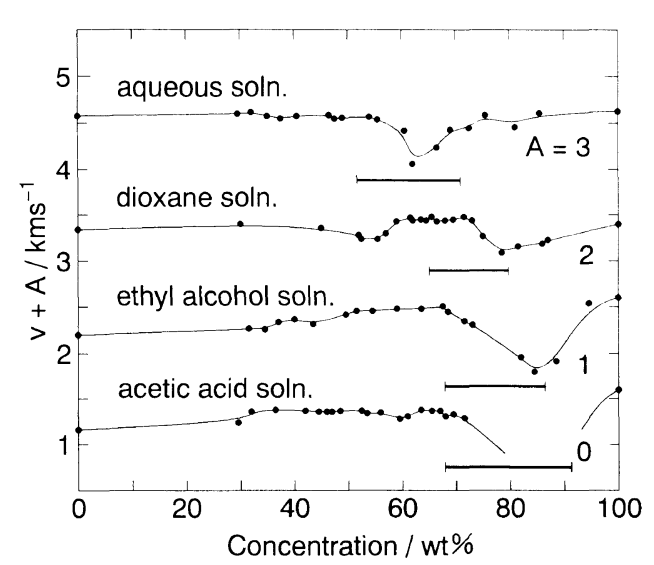

Figure 4. The variation of velocity with concentration for different solvents.

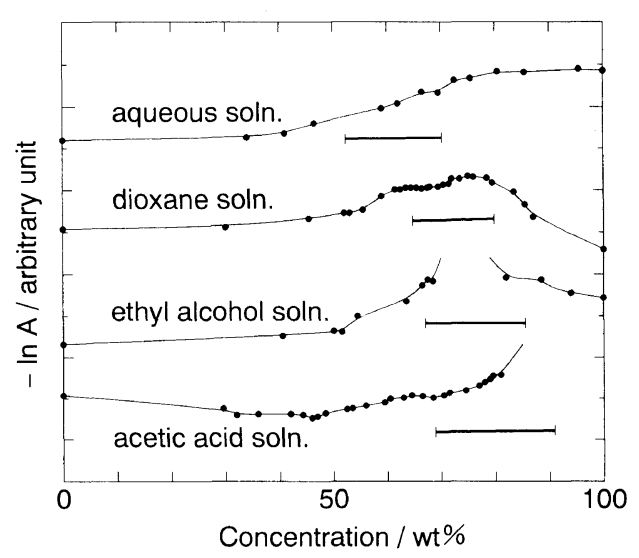

Figure 5. The variation of $-\ln A$ with concentration for different solvents.

the phase transition from isotropic to liquid crystalline phases. The anomalous behavior in the velocity and the attenuation coefficient can be referred to as Landau-Khalatnikov relaxation. ${ }^{24-27}$ It has been already found in the nematic-isotropic phase transition temperature region for low molecular weight materials. However, Landau-Khalatnikov relaxation has not been observed not only for polymers but also for lyotropic liquid crystalline materials. The present observation is the first one for lyotropic systems, so far as we know. The critical concentration at which ultrasonic variables change anomalously varied

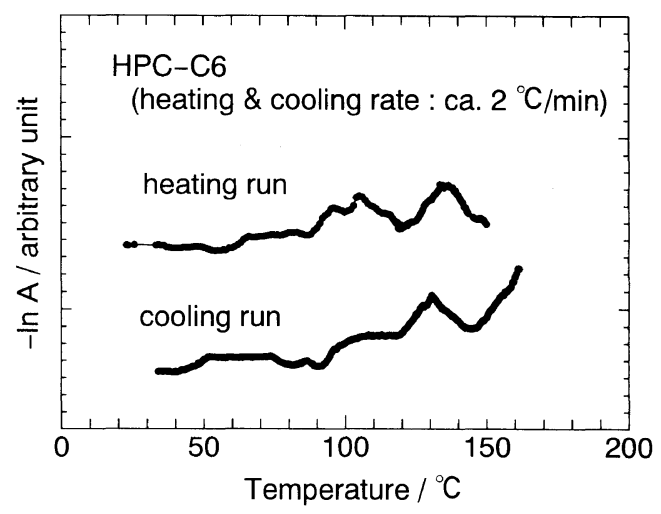

Figure 6. The variation of $-\ln A$ ( $A$ is the amplitude of sonic wave at a given distance) with temperature for HPC-C6. The heating and cooling rates are about $2 \mathrm{Cmin}^{-1}$.

with species of solvent; it became higher in the order of acetic acid, ethanol, dioxane, and water.

Another point should be noted; for HPCdioxane solutions, another small drop was clearly found on the plot of ultrasonic velocity $v s$. concentration and another small maximum on the plot of intensity $v s$. concentration. Looking carefully at the plots for other solvent systems, one can also find a similar drop or maximum. It is indicated that another kind of phase transition exists besides that from isotropic to liquid crystalline phases. The origin is not clear at present. However, it is possibly considered that some sort of molecular ordering takes place before formation of liquid crystalline phase.

\section{Temperature Dependence of Ultrasonic Prop-} erties of $\mathrm{HPC}$-C6

Ultrasonic properties of HPC-C6 were measured in the temperature region between 20 and $170^{\circ} \mathrm{C}$. Figure 6 shows the variation of $-\ln A$ with temperature. One can find more than two peaks both on heating and on cooling runs in the figure. One peak is seen at temperatures between 130 and $135^{\circ} \mathrm{C}$. This temperature range coincides to the temperature region in which a peak appeared on the DSC 
thermogram. Otherwise, the following change was observed in the same temperature range under polarized microscope; on a heating run it became fully dark in a field of vision, and on a cooling run small pieces of illumination appeared. The former indicates that liquid crystalline phase disappears perfectly and the latter that liquid crystalline phase begins to form. Thus, the peak of the ultrasonic intensity at temperatures between 130 and $135^{\circ} \mathrm{C}$ can be expected to be caused by the phase transition between liquid crystalline (maybe cholesteric) and isotropic phases.

Other peaks were found in the temperature region between 90 and $120^{\circ} \mathrm{C}$ especially on the heating run. On the other hand, neither endothermic nor exothermic peak was observed on the DSC thermogram in the same temperature region and no particular change on a polarized microscope. It may be indicated that the phase transition might be of the second kind; the detail mechanism of the transition is not clear at present. However, it seems to be noteworthy to show the following observation under a polarized microscope; the band structure $^{28,29}$ is often formed by very small but unavoidable shear force when one inserts samples between slide and covered glasses. The band structure thus formed loosened and melted in the temperature region between 90 and $120^{\circ} \mathrm{C}$. On the contrary, although no band

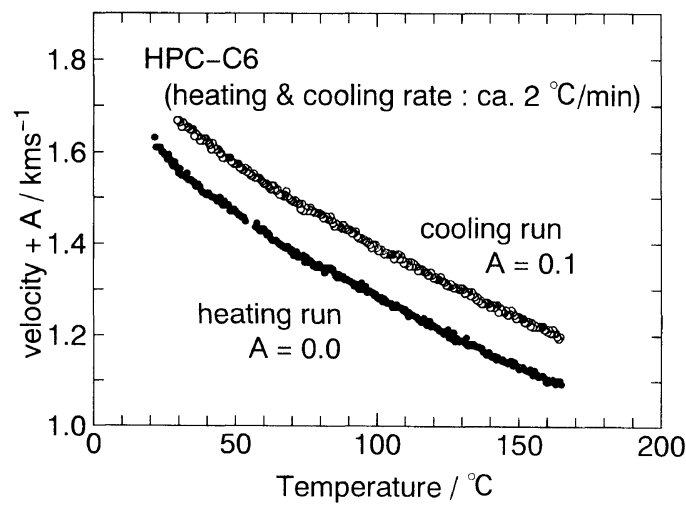

Figure 7. The variation of the ultrasonic velocity with temperature for HPC-C6. The heating and cooling rates are about $2^{\circ} \mathrm{C} \min ^{-1}$. structure was seen on the second heating run, the ultrasonic peak appeared. It is indicated that the ultrasonic peak may be associated with the change in the intermolecular state; the state may be necessary to keep a band structure, but not enough to form a band structure.

Figure 7 shows the variation of the ultrasonic velocity with temperature for HPC-C6. As is shown in the figure, no peak was observed both on heating and on cooling runs. One may find a shoulder at temperatures at which $-\ln$ $A$ takes a peak when one carefully examines the figure. However, it is unclear. It is suggested that the phase transition intensity is very small. The change may be very close to the phase transition of the second kind, in which the molecular arrangement (orientation) may mainly change.

\section{SUMMARY}

Ultrasonic properties of HPC solution were measured in the evaporation process. The phase transition of Landau-Khalatnikov type was first observed in lyotropic liquid crystalline polymer systems, so far as we know. The critical concentration varied with species of solvent and it became higher in the order of acetic acid, ethyl alcohol, dioxane, and water.

Ultrasonic properties of HPC-C6 in bulk were measured as a function of temperature. They showed at least two phase transitions: One of them is ascribable to one between liquid crystalline and isotropic phases (i.e., the phase transition of the first kind). Another phase transition may be one of the second kind, in the case in which one could not detect by conventional methods.

Thus, it was found that the ultrasonic method is very useful to study phase transition behavior.

\section{REFERENCES}

1. R. S. Werbowyj and D. G. Gray, Macromolecules, 
13, 69 (1980).

2. J. Behda, J. F. Fellers, and J. L. White, Colloid Polym. Sci., 258, 1335 (1980).

3. T. Tsutsui and R. Tanaka, Polym. J., 12, 473 (1980).

4. P. Flory, Proc. R. Soc. London, Ser.-A, 234, 73 (1956).

5. D. G. Gray, J. Appl. Polym. Sci., Appl. Polym. Symp., 37, 179 (1983).

6. R. D. Gilbert and P. A. Patton, Prog. Polym. Sci., 9, 115 (1983).

7. D. G. Gray, Faraday Discuss. Chem. Soc., 79, 257 (1985).

8. K. Shimamura, J. L. White, and J. F. Fellers, J. Appl. Polym. Sci., 26, 2165 (1981).

9. S.-L. Tseng, A. Valente, and D. G. Gray, Macromolecules, 14, 715 (1981).

10. G. Charlet and D. G. Gray, Macromolecules, 20, 33 (1987).

11. A. M. Ritcey and D. G. Gray, Macromolecules, 21, 1251 (1988).

12. S. N. Bhadani and D. G. Gray, Makromol. Chem., Rapid Commun., 3, 449 (1982).

13. T. Yamagishi, Doctor Thesis, Kyoto University, 1989.

14. L. D. Landau and E. M. Lifshitz, "Statistical Physics," Pergamon Press, 1980.
15. K. Okano, "Ekisho" Japanese, K. Okano and S. Kobayashi, Ed., Baifukan, Tokyo, 1985.

16. A. B. Bhatia, "Ultrasonic Absorption," Oxford University Press, London, 1967.

17. C. Robinson, Trans. Faraday Soc., 52, 571 (1956).

18. C. Robinson, Mol. Cryst., 1, 467 (1966).

19. H1. de Vries, Acta Cryst., 4, 219 (1951).

20. M. Horio, E. Kamei, and Y. Uchimura, Jpn. J. Rheol., 13, 25 (1985).

21. C. J. Malm, J. W. Mench, D. L. Kendall, and G. D. Hiatt, Ind. Eng. Chem., 43, 684 (1951).

22. P. K. Chatterjee and C. W. Conrad, J. Appl. Polym. Sci., 11, 1387 (1967).

23. F. F. L. Ho, R. R. Kohler, and G. A. Ward, Analytical Chem., 44, 178 (1972).

24. L. D. Landau and I. M. Khalatnikov, Dokl. Akad. Nauk USSR, 96, 469 (1954).

25. M. Fixman, J. Chem. Phys., 36, 1961 (1962).

26. H. Imura and K. Okano, Chem. Phys. Lett., 19, 387 (1973).

27. S. Bhattacharya, B. K. Sarma, and J. B. Ketterson, Phys. Rev. B, 23, 2397 (1981)

28. J. Takahashi, K. Shibata, S. Nomura, and M. Kurokawa, Sen-i Gakkaishi (in Japanese), 38, T-375 (1982).

29. P. Navard, J. Polym. Sci., Phys. Ed., 24, 435 (1986). 